Musées, Patrimoine et Culture scientifiques et techniques

142 | 2012

juillet-août 2012

\title{
Les labels du Patrimoine culturel
}

\section{Marion Roux-Durand}

URL : http://journals.openedition.org/ocim/1095

DOI : 10.4000/ocim.1095

ISSN : 2108-646X

\section{Éditeur}

OCIM

Édition imprimée

Date de publication : 1 juillet 2012

Pagination : 28-37

ISSN : 0994-1908

Référence électronique

Marion Roux-Durand, « Les labels du Patrimoine culturel », La Lettre de I'OCIM [En ligne], 142 | 2012,

mis en ligne le 01 juillet 2014, consulté le 19 avril 2019. URL : http://journals.openedition.org/

ocim/1095; DOI : 10.4000/ocim.1095 


\section{Les labels du Patrimoine culturel}

\section{Marion Roux-Durand *}

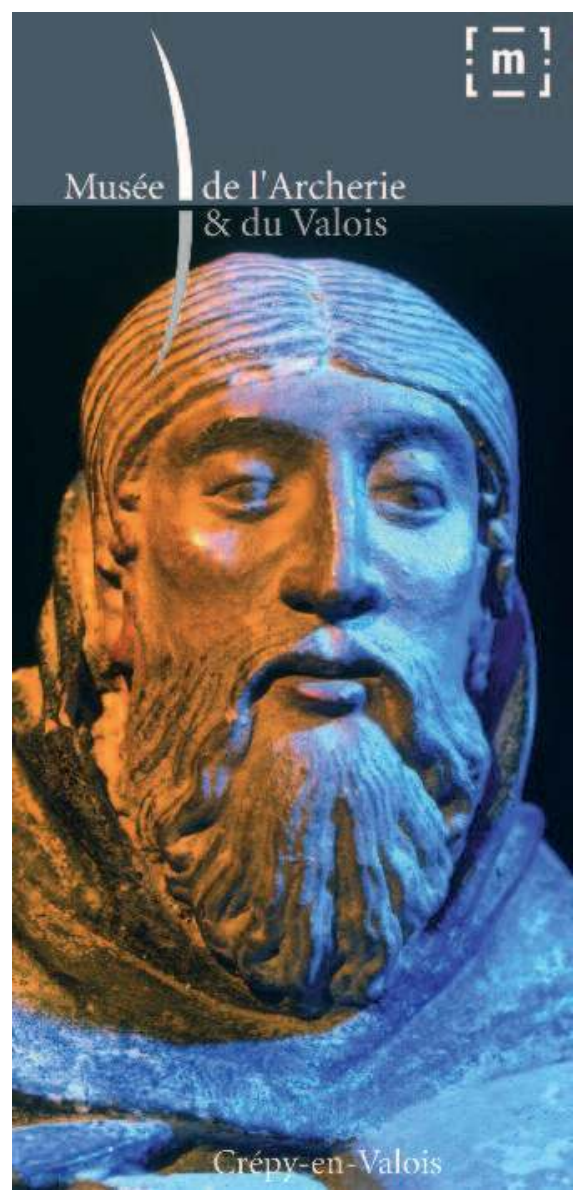

Sur les différents supports de communication employés par les musées, le logotype symbolisant le label « Musée de France » apparaît comme une caution scientifique reconnue du public et des professionnels. ๑) Ville de Crépy-en-Valois

* Marion Roux-Durand est attachée de conservation du patrimoine, responsable du musée de l'Archerie et du Valois à Crépy-en-Valois marion.roux@mairie-crepy-en-valois.fr
L'auteur propose ici d'identifier les labels culturels, de comprendre la démarche de labellisation, d'évaluer - à la lueur des enjeux actuels de la sphère patrimoniale les principes de mise en œuvre dans de telles initiatives pour en définitive se demander si le nombre croissant des labels n'entraînera pas à terme une forme de redéfinition des musées.

Les labels, dans le domaine de la Culture, ne semblent souvent être abordés que par l'omniprésence de l'appellation « Musée de France », accaparant l'attention des spécialistes comme celle des professionnels des musées. Ce vide documentaire a précisément motivé notre réflexion qui a pris place dans le cadre d'un mémoire de recherche en muséologie à l'École du Louvre. Le recensement de ces labels déjà complexe ne saurait être ici exhaustif.

\section{Qu'est-ce qu'un label culturel ?}

Question cruciale, s'il en est, à laquelle il semble difficile de répondre, même pour des professionnels directement impliqués. Mieux connus dans les sphères commerciales, les labels ont peu à peu gagné de nouveaux champs d'application : celui du tourisme et, plus récemment, celui de la culture. La multiplicité des labels issus de la sphère commerciale ou ceux relevant d'autres ministères sont légion, et ne pourraient être tous évoqués, sinon à titre de comparaison. En effet, les points communs pouvant être établis entre ces différents domaines révèlent une définition relativement vague et, malheureusement, insatisfaisante. 
Une recherche étymologique permet dans ce cas de révéler toute la polysémie du terme : dès le MoyenÂge, le terme de «lambel », synonyme de «blason », affirme l'importance des notions de visibilité mais aussi d'identité dans la compréhension du mot. Réapparu en France au XVIIe siècle, après avoir été approprié par la langue anglaise, le terme nous revient comme désignant une étiquette, une bande de papier collée sur un produit donnant des indications quant à son appellation, son origine, sa composition et son usage. Une définition qui n'est pas sans rappeler que dans les pays anglo-saxons, «label » désigne encore ce que nous appelons désormais le cartel d'une œuvre.

Lacception la plus courante demeure toutefois, et sans conteste, porteuse d'un sens mercantile, tendant à assimiler le label à la marque déposée qui garantit la qualité voire la conformité d'un produit avec des normes de fabrication préétablies. Cette connotation commerciale qui, a priori, est en complète opposition avec la déontologie culturelle d'un musée se refusant tout profit, crée un paradoxe d'autant plus instructif que leur monopole est détenu par l'administration du ministère de la Culture et de la Communication.

"Certificat de conformité », "signe de reconnaissance », il y a tout lieu de penser que le label joue le rôle d'identification pour le «produit » qui l'arbore, immédiatement reconnaissable par le logotype qui lui est associé. Littéralement, le label est un symbole, puisque sa possession implique une démarche et un engagement de la part de ceux qui souhaitent s'y rallier : porteur d'une identité, le label définit et reconnaît ainsi les qualités d'une action, d'un produit, les distinguant par là-même d'un ensemble jugé commun. Bien souvent, on peut remarquer que la définition de « label » se fait en négatif, par comparaison avec des notions qui nous sont mieux connues : il se distingue ainsi d'une marque déposée, d'une appellation, mais aussi d'un prix que l'on reçoit à l'issue d'un concours.

Cette étude a pu révéler en outre que la signification du mot « label » est parfois fluctuante, apposée sur des démarches variées, au point qu'il semble que chaque initiative livre sa propre compréhension du terme. Nous avons de ce fait choisi de retenir comme critère de définition que le label est la marque d'une contractualisation, s'apparentant à un signe de ralliement fédérateur d'un réseau d'acteurs dont témoigne la mise en place de logotypes et de chartes graphiques conçus à cet effet. Il est ainsi porteur d'un ensemble d'informations, pour qui sait le lire, s'inscrivant en même temps dans un système de communication complexe.

\section{Labels mondiaux, labels européens}

Avant même d'aborder les labels inscrits dans la législation française, il convient de considérer d'autres mesures de reconnaissance qui bénéficient d'une large considération auprès des responsables culturels français, européens et internationaux, au point qu'en France, l'État et les collectivités territoriales, ne puissent faire autrement que de les inclure dans leurs actions. Ainsi en est-il des labels délivrés par l'UNESCO tels que «Patrimoine mondial », « Patrimoine immatériel » ou « Mémoire du Monde », qui sont peu à peu devenus synonymes de l'identité culturelle mondiale, des symboles très convoités puisqu'à l'heure actuelle 936 sites sont inscrits. Les labels de l'UNESCO n'incarnent pourtant qu'une petite partie de l'action de l'organisation mondiale, la plus grande part de ses activités se concentrant plutôt sur la mise en place de

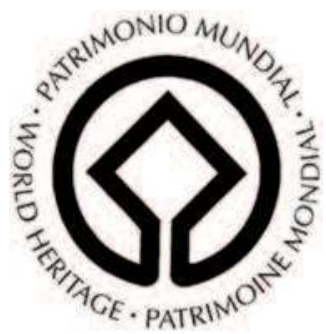
conventions, dont l'objectif est de mettre en lumière la nécessité d'une protection imminente de patrimoines jugés en péril.

Le rôle essentiellement prescriptif de l'UNESCO rejoint de ce point de vue les initiatives d'autres organisations plus spécialisées telles que l'ICCROM, l'ICOMOS ou l'ICOM, qui proposent leur expertise tout en établissant des normes supranationales dans les domaines de la conservation et de la restauration d'œuvres d'art, des monuments et des sites mais aussi des musées. Bien qu'on ne puisse pas à proprement parler de « label » concernant ces organismes, on peut observer des similitudes de fonctionnement. L'ICOM qui a pour formule "Les musées, les professionnels des musées, le label d'excellence » ne reconnaît que les institutions répondant aux exigences déontologiques et à la définition qu'il propose. L'ICCROM et l'ICOMOS se positionnent également comme les garants d'une excellence qu'ils partagent avec leurs membres, fédérant ainsi tout un réseau d'établissements pour qui l'adhésion à ces normes constitue en soi une labellisation.

Le contexte est relativement différent en ce qui concerne les labels culturels délivrés par la Conseil de l'Europe notamment parce qu'une fois ratifiées, ces directives doivent être transposées dans les systèmes législatifs respectifs de chaque pays membre. D'après un sondage réalisé par IPSOS en 2007, «l'Europe culturelle semble être aujourd'hui une réalité véritablement 
ressentie par une bonne part des citoyens de l'Union Européenne » (1). Ce sondage révèle en partie les résultats de plusieurs initiatives menées depuis plus de trente ans visant à construire ce sentiment d'appartenance. Dès 1987, le Conseil de l'Europe lançait les «Itinéraires culturels ", avec pour objectif "de démontrer, à travers le voyage dans l'espace et dans le temps, que le patrimoine des différents pays d'Europe et leur culture contribuent an patrimoine culturel commun». Un accord élargi contribua par la suite à renforcer les coopérations en matière culturelle, en insistant sur les éléments symboliques d'unité, d'histoire et de valeurs européennes. Rejoignant cette volonté, le label «Patrimoine européen » fut lancé en 2007, s'adressant aux sites ayant joué un rôle important dans le processus d'intégration euro-

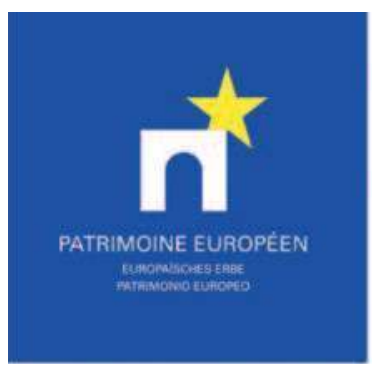
péenne. Un troisième exemple, que nous fournit la création en 2008 du label «Évènement culturel de l'Europe ", permet de comprendre le rôle que le Conseil de l'Europe attribue à ce type de reconnaissance : en s'inscrivant dans les valeurs fondamentales de l'Union européenne, les labels sont chargés de construire des références communes et sont employés comme des promoteurs d'une identité culturelle encore en construction.

\section{Les labels du ministère de la Culture et de la Communication}

\section{Un label pour protéger}

L'apparition de labels spécifiques au domaine patrimonial français remonte aux années 1980, bien que le classement et l'inscription au titre des Monuments Historiques puissent à certains égards apparaître comme des précurseurs dans ce domaine. Initié en 1985 avec le label «Ville et Pays d'art et d'histoire », le mouvement de labellisation ne semble réellement se répandre qu'à partir des années 2000, à commencer par l'incontournable label « Musée de France ».

Avant que ne soit promulguée la loi du 4 janvier 2001, le projet d'un texte venant abroger l'ordonnance provisoire de 1945 faisait déjà débat. Dès 1993, une proposition de loi, rapportée par La Lettre des Musées de France, précisait : "le principe général du texte de loi est celui d'une politique contractuelle, fondée sur la liberté laissée aux collectivités gestionnaires de musées d'adhérer à un certain nombre de règles essentielles au bon fonctionnement des musées » (2). La question était alors de savoir quels établissements méritaient l'appellation de «musée », mais également quelles collections devaient être protégées et selon quels moyens. Le fait que l'État se prévale du rôle de juge en matière de patrimoine national avait alors hérissé les collectivités locales, tout comme il avait inquiété les collectionneurs privés. Près de vingt ans plus tard, les réflexions aboutirent à un projet plus souple, octroyant d'office cette appellation aux anciens musées classés et contrôlés ainsi qu'aux établissements nationaux, laissant en revanche la possibilité de faire la demande de ce label aux autres propriétaires de collections, sous certaines conditions.

L'octroi du label " Musée de France », qui comme son retrait est détaillé dans le Code du Patrimoine, nous révèle un dispositif très protecteur pour les collections, envisageant désormais un statut pour les dépôts et les prêts d'œuvres. Autre mesure essentielle, la loi précise les missions fondamentales devant être assurées par l'établissement détenteur du label, soulignant le caractère professionnel de ces activités et permettant par là même de distinguer «les lieux de la mémoire collective des échoppes commerciales usant et abusant du terme générique de musée » (3). Bien que cette loi soit aujourd'hui bien assimilée, le label demeure encore contesté par bien des professionnels de musées qui préfèrent le désigner comme une appellation. Le doublement de la loi par un label apparaît pour certains quelque peu incompréhensible, allant jusqu'à amoindrir les dispositions législatives selon ceux qui auraient préféré une protection du statut même de «musée ». Peut-on y voir une manière de hiérarchiser entre eux les musées publics, de les mettre en concurrence afin d'accélérer la réalisation d'objectifs et, à terme, d'homogénéiser le niveau de qualité des établissements muséaux ? Nous pouvons pour le moins remarquer que le label « Musée de France » est un outil de normalisation pour le ministère de la Culture et de la Communication, comparable en cela à l'accreditation program de l'American Association of Museums.

\section{Des labels pour sensibiliser}

Les autres labels du ministère de la Culture et de la Communication étonnent par leur grande diversité. Le premier à avoir intégré ce corpus est celui de "Ville et Pays d'art et d'histoire », créé en 1985 par Max Querrien, alors directeur de la Caisse nationale des Monuments Historiques et des Sites. Il se présente comme une convention signée entre le ministère et les acteurs territoriaux, affichant comme enjeu principal la sensibilisation et la préservation du cadre 
de vie, bien que les volets financiers et touristiques soient également essentiels dans cette démarche de valorisation. En contrepartie du label, les termes de

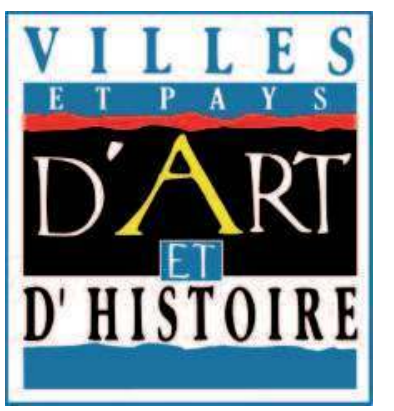

l'échange engagent les collectivités à mobiliser des moyens humains et matériels pour lui donner un sens en fonction des considérations et des priorités locales. Ainsi, des structures d'accueil touristique sont mises en place afin de protéger un patrimoine, diffuser sa connaissance tout en augmentant l'attractivité d'un territoire.

Cette démarche de valorisation qui s'inscrit pleinement dans la notion moderne de projet culturel s'est peu à peu étendue à de nouveaux patrimoines, permettant à chacun de mettre en place sa propre politique de développement local. C'est dans ce même but que le label « Patrimoine du XXe siècle » a vu le jour à l'aube des années 2000, avec pour objectif de sensibiliser le grand public à une architecture moderne qui lui est souvent méconnue. À la différence du patrimoine industriel qui, une fois inscrit sur les registres de l'Inventaire régional, peut recevoir les mesures d'inscription et de classement, et contrairement au label « Musée de France » qui fournit une législation adaptée aux collections, cette reconnaissance n'implique pas de protection, même si dans les faits elle en constitue souvent le préalable. Cette mesure est en effet présentée comme une alternative aux lourdes procédures de préservation existantes, justifiée en outre par le manque de recul actuel qui rend difficiles les choix de protection. La mise en place de labels cherche ainsi à s'adapter à une ouverture de la définition classique de la culture mais c'est aussi, semble-t-il, un moyen de cerner les spécificités de chaque patrimoine.

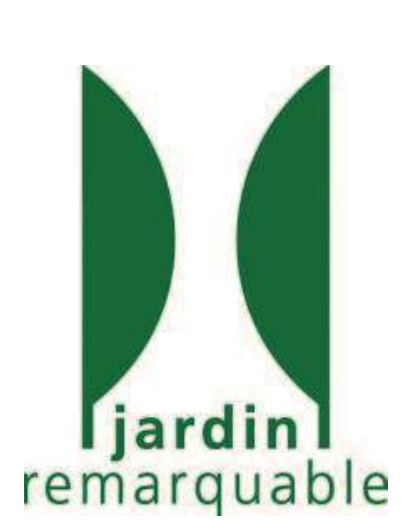

Cela explique la grande diversité de statuts juridiques et de clauses quimposent les labels, à l'exemple des «Jardins remarquables », créés par circulaire le 17 février 2004, dont la labellisation n'a une durée effective que de cinq ans seulement et s'adresse également aux propriétaires réalisant des bénéfices commerciaux. La démarche de labellisation repose principalement sur une relation d'échange, le demandeur pouvant bénéficier d'un soutien scientifique et technique en contrepartie de son engagement à respecter des critères de conformité fixés par le détenteur du label. Une évolution de cette pratique de contractualisation est toutefois à noter : certains labels offrent la possibilité d'apporter un soutien financier aux établissements qui en font la demande, sous réserve bien entendu de remplir les conditions imposées par les organismes détenteurs, publics ou privés.

\section{Des labels pour valoriser}

Le label de la Fondation du Patrimoine agit ainsi par le biais de subventions accordées aux propriétaires de biens immobiliers dignes d'intérêt mais non protégés par l'État. L'idée sous-jacente dès sa création en 1996 était bel et bien de mettre en place un trust apportant son aide financière et technique afin de "promouvoir la connaissance, la conservation et la mise en valeur du patrimoine national ». Ces dispositions permettent de ce fait de mettre en valeur des monuments qui ne sont pas jugés «prioritaires » par le ministère et de les intégrer à un réseau, à l'exemple du patrimoine rural qui demeure bien souvent à la charge exclusive de son propriétaire. La Fondation du Patrimoine s'inspire ainsi largement de l'exemple anglais et de l'organisation non gouvernementale English Heritage qui rempli ce rôle de protecteur de la mémoire collective dont nous estimons en France qu'il est du ressort de la puissance publique. Le label est dans ce cas le signe visible d'une adaptation à une nouvelle réalité économique, comme le souligne le préambule du Livre blanc des Musées de France : «Le propriétaire et éventuellement d'autres partenaires tels que l'État, des collectivités, des entreprises ou des personnes privées, apportent des ressources complémentaires sous la forme de subventions d'équilibre ou de dotations (mécénat) sans lesquelles le musée ne pourrait remplir ses missions de service public».

De ce fait, aucun établissement culturel ne peut fonctionner sans soutien financier, et c'est en partie dans ce contexte que fut créé le label « Exposition d'intérêt national », expérimenté dès 1999 par le ministère de la Culture et de la Communication et qui a pour but de primer chaque année les expositions les plus remarquables pour leur intérêt scientifique, leurs efforts en terme de médiation culturelle et leur caractère innovant. Cette aide spécifique dirigée vers les musées de région a permis, en 2011 , de doter les seize musées primés de subventions allant de 10000 à 50000 euros. Plusieurs remarques peuvent dès 
La question se pose de savoir quelle différence distingue un label d'un prix de récompense, mais aussi de comprendre pourquoi, depuis quelques années, le nombre de labels s'est multiplié, au point parfois de concurrencer des initiatives déjà existantes. La différence principale semble résider dans le fait que le label s'inscrit dans une durée, il est efficient sur plusieurs années et répond à des caractéristiques précises, à une grille de normes préétablies. De manière différente, le prix est attribué par un jury (et non un comité d'experts) qui, régulièrement, récompense une initiative globale jugée innovante.

Ainsi, le label « Tourisme et Handicap » est attribué en reconnaissance de l'adaptation d'un établissement à une déficience spécifique, selon une liste de critères définie à l'avance, tandis que le prix «Musée pour Tous » vient récompenser une démarche qui ne récompense pas seulement l'aménagement du bâti mais aussi le traitement des informations, l'accueil du public handicapé et les efforts déployés pour que tous les publics bénéficient ensemble de ces transformations.
En outre, l'intérêt du prix est de susciter une émulation parmi les participants, créant un contexte de compétition propre à valoriser ce type d'initiative auprès des élus. Il est possible que ce soit justement pour éviter l'amalgame avec une mise en concurrence que les initiateurs des « Expositions d'intérêt national » ont choisi de les concrétiser sous le statut du label plutôt que d'un prix. Il n'en demeure pas moins que cette démarche s'apparente dans les faits plus à un concours qu'à une accréditation. Les labels ne seraient-ils finalement qu'un abus de langage ?

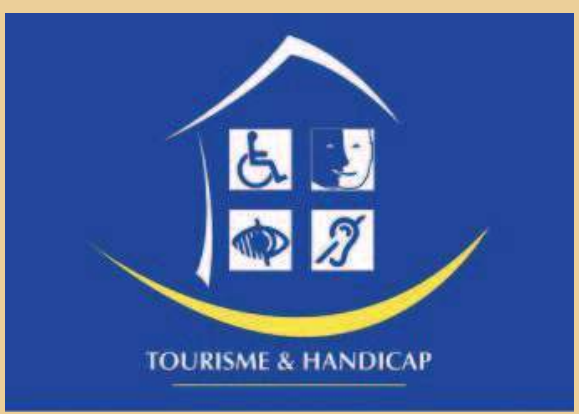

lors être émises, notamment quant à la nature de ce label : tout d'abord, pourquoi créer un label et non simplement un prix venant récompenser les efforts d'une institution ? Choisir le label est en effet étonnant si l'on considère la courte durée de son attribution. Par ailleurs, le montant des subventions octroyées, s'il semble conséquent aux yeux du grand public, apparaît bien en deçà des réels coûts de montage d'une exposition. On peut dès lors se demander si ce label ne poursuit pas d'autres buts : plus qu'un véritable soutien, il constitue en effet un moyen important de communication, et pour l'État et pour les établissements primés, qui espèrent accroître leur attractivité auprès du public. Ce que l'on pourrait redouter à terme serait une mise en concurrence généralisée des musées qui, avec les rétrécissements budgétaires, devraient justifier un soutien financier supplémentaire.

\section{La démarche de labellisation: enjeux et limites}

Dans un contexte où la demande de patrimoine est très forte, les institutions muséales doivent défendre leur singularité et la qualité de leur offre. Qu'ils le veuillent ou non, les musées s'inscrivent désormais dans un «marché culturel » dans lequel la labellisation et le système de marques sont des moyens mis en œuvre depuis longtemps déjà, notamment par les professionnels du tourisme. Les labels émis par le ministère du Tourisme, mais aussi ceux déployés par les professionnels, les associations et les fédérations constituent des atouts communicationnels indéniables dans la promotion d'une offre et de l'attractivité d'un site, comme l'ont aussi bien compris les collectivités territoriales. À cela s'ajoute la création de labels « mixtes », qui ciblent leur clientèle en couplant labels environnementaux et patrimoniaux. Le secteur touristique propose ainsi des prestations de plus en plus adaptées, centrées sur les attentes du public et venant concurrencer directement les musées sur le terrain de l'offre culturelle.

Pour beaucoup, à l'instar de Dominique Audrerie, «le label est ainsi un argument de notoriété, dont se servent les marchands de patrimoine pour séduire un public et attirer des visiteurs » (4). Il en résulte, selon lui, une forte ambiguïté dans l'usage qui est fait du mot « label » dont le sens premier ne semble pas correspondre à un bien patrimonial, au contraire. Il n'en demeure pas moins que dans ce contexte, les politiques culturelles cherchent elles aussi à innover, se positionnant parfois même au cour de contradictions avec les 
objectifs initiaux des musées. La firme Guggenheim offre un exemple emblématique de ce que peuvent devenir les établissements patrimoniaux en adoptant la logique de l'ingénierie culturelle, structurés en « maison-mère » et en filiales, selon un procédé qualifié par André Gob de «mimétisme entrepreneurial » (5).

Bien qu'encore réticents, les grands musées occidentaux savent qu'ils sont aussi devenus des outils de concurrence commerciale, économique et touristique. La polémique qui entoure la création d'une succursale du Louvre à Abou Dhabi souligne ce malaise : au-delà même des termes financiers qui président cette tractation avec les Émirats arabes unis, la question de l'exportation de l'appellation « Louvre » pose un problème éthique car, contrairement à la marque de la fondation Guggenheim, il s'agit d'une propriété publique dont on ne peut disposer comme d'un bien familial. Cet exemple extrême illustre la nuance ténue qui existe entre le « label » et la «marque », le premier préfigurant peut-être une évolution vers la seconde.

\section{La nécessité d'un réseau}

Les exemples figurant l'insertion progressive de certains musées dans une "économie de limmatériel » révèlent les besoins croissants des institutions patrimoniales afin de valoriser leurs collections. On peut de ce fait voir dans la mise en place de labels, en particulier dans la volonté d'instaurer entre les établissements labellisés une forme de coopération, un moyen de pallier l'absence de ressources nouvelles en encourageant une certaine forme de mutualisation. Plus nombreuses dans le domaine des arts du spectacle, de telles initiatives existent aussi pour les musées, que ce soit par la conception commune d'une exposition temporaire, l'alimentation d'une base de données mutualisée, et la volonté de mise en réseau est d'ailleurs affirmée par chaque décret ministériel portant création d'un nouveau label. Il convient toutefois de nuancer la portée effective de cette orientation, en remarquant par exemple que, malgré le rôle conséquent joué par les DRAC, les partenariats entre " Musées de France » sont plutôt le fruit de volontés particulières que d'une politique active de mise en réseau dépassant le simple recensement des établissements labellisés.

À l'inverse, des réseaux sans labels existent, à l'exemple de la Fédération des écomusées et des musées de société qui a su moderniser son approche patrimoniale en intégrant les musées de société et les centres d'interprétation, et a fait de la coopération une dimension essentielle, notamment pour des structures qui ne peuvent s'appuyer que sur de faibles subventions.

\section{L'enjeu de visibilité}

Signes de prestige et de reconnaissance, les labels semblent créer une émulation entre établissements et sites patrimoniaux afin de hausser les exigences de l'offre culturelle. Les dispositions qui les accompagnent, symbolisées par des logotypes, agissent donc

\section{Les associations, moteurs de lobellisation}

Les initiatives privées peuvent constituer une source d'inspiration pour les pouvoirs publics, mais elles permettent également d'ouvrir les établissements patrimoniaux à de nouvelles préoccupations de société. En effet, les 150000 associations culturelles, qui représentent près d'un cinquième du nombre total d'associations en France, jouent un rôle puissant dans le développement du patrimoine national, ne serait-ce que parce qu'elles bénéficient du soutien des collectivités territoriales et du ministère, qui leur accorde plus de subventions qu'à toute autre forme d'organisation.

Un des meilleurs exemples de cette impulsion associative est celui de «Tourisme et Handicap » qui a mis en place un label reconnu par les pouvoirs publics permettant de délivrer des informations précises et objectives sur l'accessibilité des sites culturels et des équipements touristiques, en prenant en compte tous les types de handicaps identifiés chacun par un pictogramme. Tout en œuvrant pour un meilleur accès aux structures patrimoniales, le label permet aux professionnels qui l'arborent de faire valoir un avantage concurrentiel important à l'échelle nationale, européenne, voire internationale. Preuve de réussite pour l'association, le secrétariat d'État au Tourisme et le secrétariat en charge de la Famille et de la Solidarité ont publié le 22 septembre 2010 un appel à candidature pour le recrutement de territoires pilotes d'un nouveau label appelé «Destination pour tous ». « Tourisme et Handicap » a ainsi trouvé un réel soutien dans l'action publique, bien que la gestion du réseau lui soit encore pour une grande part déléguée. 


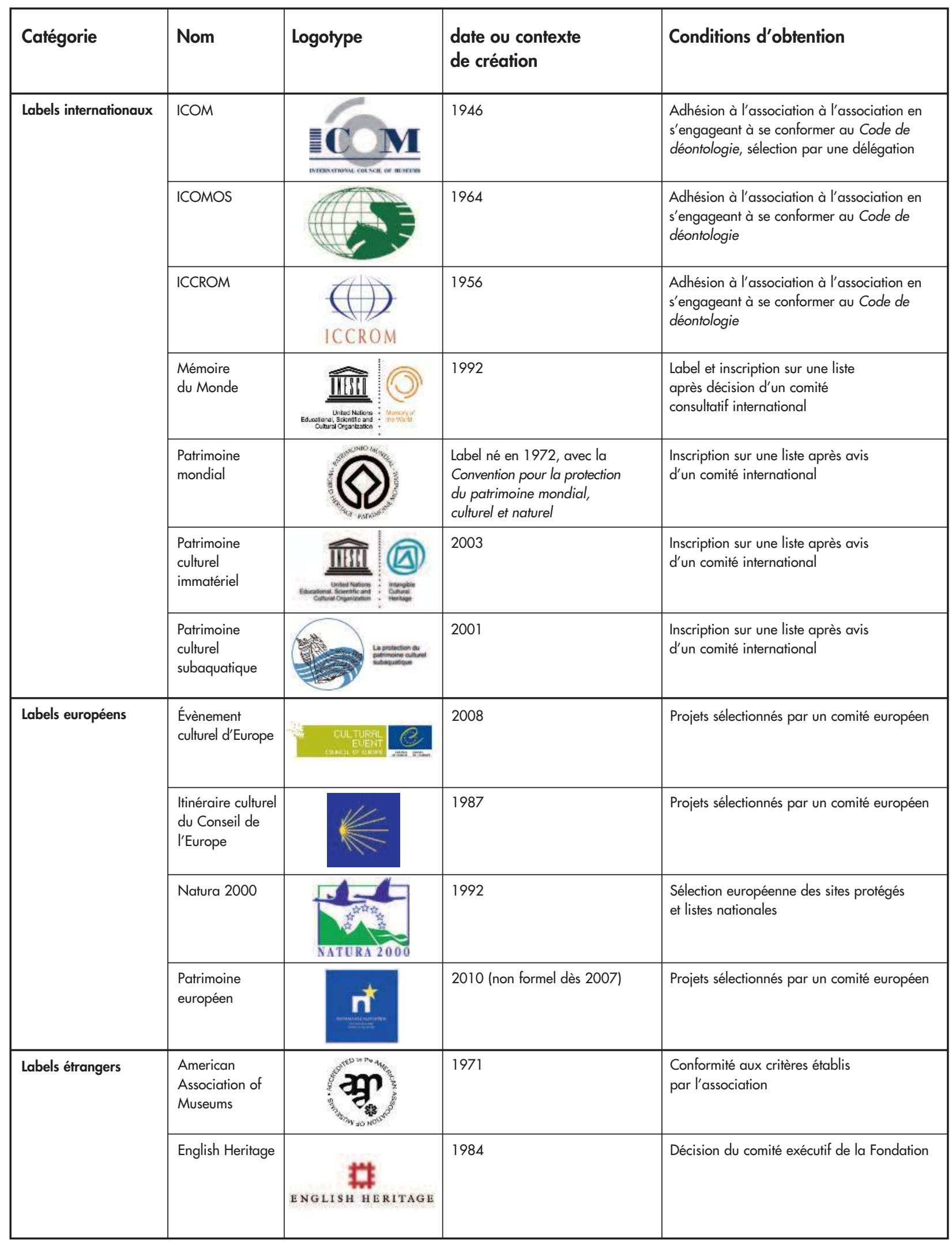




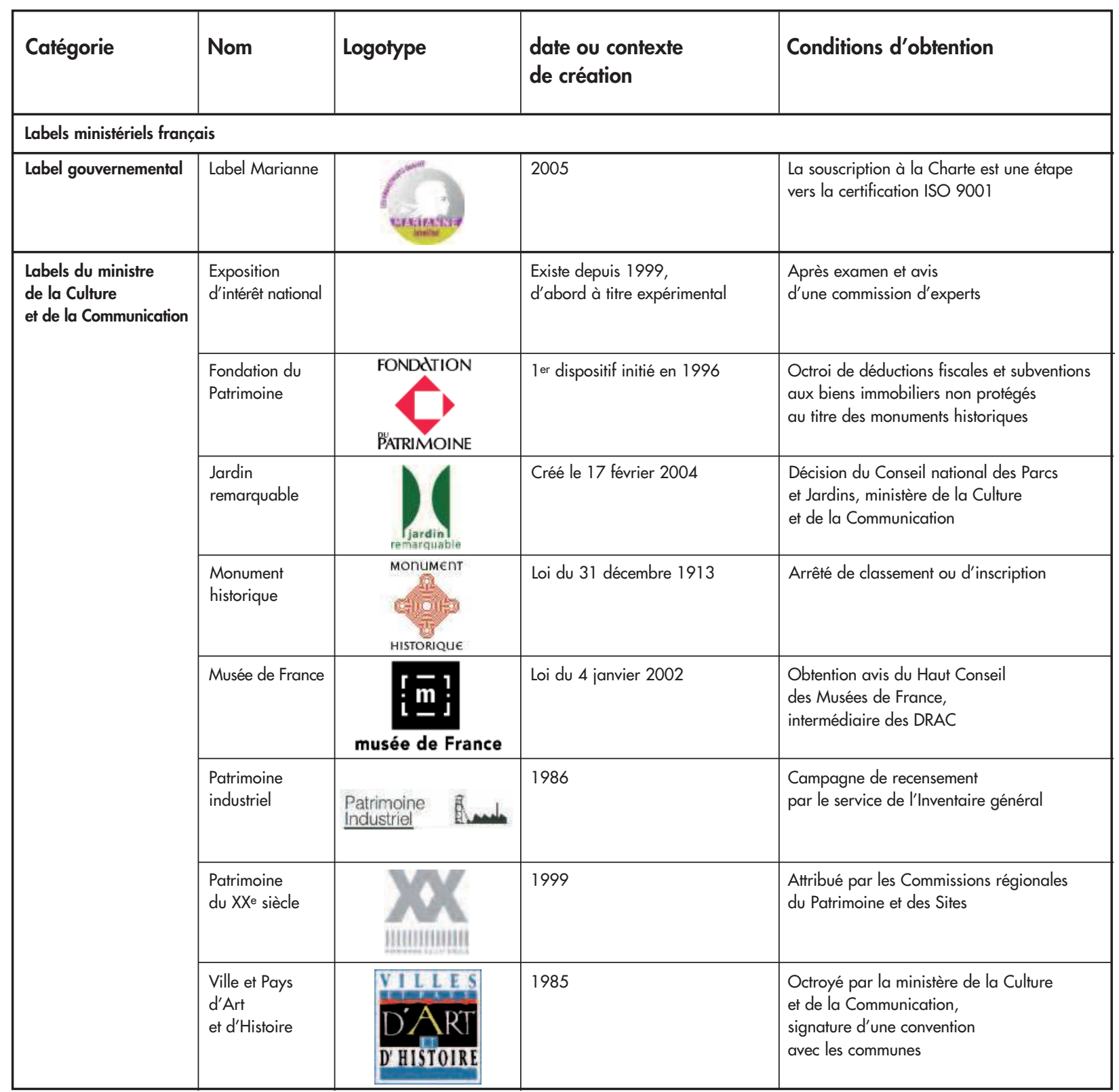

Tableau des labels évoqués dans cet article 
aussi comme des outils de communication, pouvant s'intégrer dans une démarche de marketing destinée à promouvoir un établissement, une démarche, un réseau culturel. C'est ce qu'ont compris les collectivités territoriales qui bien souvent usent de la notoriété d'un site labellisé afin de développer l'identité d'un territoire, mettant parfois en place son propre système de labellisation pour générer une cohésion locale.

Il paraît cependant étonnant que la communication au niveau ministériel demeure relativement faible, les professionnels n'étant pas toujours informés des dispositions nouvelles et de leur possibilité d'y adhérer. Plus surprenante encore est la méconnaissance du grand public qui n’identifie pas forcément l'origine ou l'implication des labels officiels, qui pourraient pourtant agir telles des garanties de haute qualité voire, pour les plus avertis, comme un argument supplémentaire de fréquentation. Ce manque de visibilité nous conduit à nous interroger sur les catégories de personnes auxquelles les labels s'adressent.
Les labels sont-ils vraiment faits pour le visiteur? Il n'existe aujourd'hui aucune étude recensant l'impact de telles initiatives sur le public ni de démarche de suivi pour les labels les plus anciens, contrairement au secteur touristique qui a coutume de mener ce type d'enquête.

\section{Peut-on parler d'une redéfinition des institutions patrimoniales?}

Si l'emploi de labels n'impose pas directement une refonte globale du fonctionnement des établissements culturels, on peut constater qu'il instaure tout du moins la prise en compte de nouveaux domaines. À l'instar du ministère de l'Enseignement supérieur et de la Recherche - qui, dès 2008, a mis en place pour les centres de culture scientifique, technique et industrielle le label

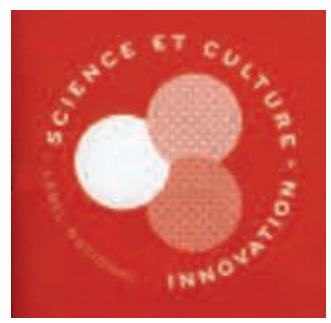

\section{Une certification de plus en plus recherchée}

Les labels patrimoniaux semblent refléter de nouvelles préoccupations envers les publics, qu'ils soient envisagés en termes de clientèle ou en tant que visiteurs auxquels les institutions publiques doivent un service. Il est intéressant de noter que la labellisation, en dehors du cas particulier que constitue le label «Musée de France », porte principalement sur les aspects visibles de l'établissement patrimonial, ils sont en quelque sorte un moyen de guider le regard en désignant ce qui fait partie du patrimoine, actualisant un domaine en perpétuelle évolution. Plus encore, les différents labels forment un répertoire d'initiatives agréées par le ministère de la Culture et de la Communication : ils sont investis d'un pourvoir efficace octroyant une reconnaissance officielle, s'apparentant à une forme de certification.

Coup de jeune ou coup de bluff ? Les labels, qui sont dans une certaine mesure des instruments d'évaluation dans le sens où ils distinguent entre eux différents établissements, ne portent en réalité que très peu sur des pratiques concrètes, l'efficacité ou la valeur de tel ou tel pôle d'activité dans le fonctionnement d'une institution. Ils entérinent des démarches existantes et ne s'inscrivent qu'a posteriori dans le mouvement de modernisation qu'engagent les établissements culturels. En cela, ils sont moins un instrument de mesure qu'une authentification par l'État.
D'autres critères ont cependant vu le jour, mis en place par des agences officielles de normalisation telles que l'AFNOR ou la COFRAC, auxquels de plus en plus de collectivités territoriales et de musées font appel pour certifier leur bonne administration. Pourrait-on à l'avenir imaginer des labels portant sur les activités vitales d'un musée, permettant par exemple de garantir l'excellence de méthodes de conservation préventive, l'expérience de médiations réussies ou l'inventaire exhaustif de collections ? Ce type de garantie de bon fonctionnement est déjà à l'œuvre dans les administrations publiques, à l'exemple de la charte Marianne devenue label Marianne, qui sanctionne une politique d'accueil dans les services de l'État.

Et si, à terme, les labels se convertissaient en indicateurs de performance pour les institutions muséales, ne devraient-ils pas être octroyés par ceux à qui ils s'adressent, à savoir les visiteurs ? Une coopération plus étroite entre les musées et leurs publics est déjà source de réflexion pour nombre de ces établissements, et notamment à l'étranger. Des expériences concluantes si on se réfère à l'ouvrage de $\mathrm{Ni}$ na Simon, The participatory museum. 
«Science et Culture, Innovation »- les labels de la Culture semblent être des moyens pour établir des passerelles transdisciplinaires qui enrichissent et renouvellent les discours portant sur le patrimoine national.

En fin de compte, la multiplicité de ces labels révèle des démarches et des finalités parfois fort variées, qui suscitent bien plus de questions qu'elles n'apportent de réponses. Quelques perspectives d'évolution sont toutefois perceptibles, à commencer par une hiérarchisation croissante entre musées, distinguant les institutions capables de s'inscrire dans un marché international et différenciant les structures concurrentielles à l'échelle nationale ou territoriale. Par ailleurs, la recherche de partenariats et de nouvelles formes de coopération incite de plus en plus les acteurs à coordonner leurs démarches, invitant les établissements patrimoniaux à devenir facteurs de développement économique mais aussi social. Ces orientations induisent de nouveaux paramètres qui, sans abolir la définition classique des musées, les obligent à se repositionner et à intégrer les attentes dont ils sont désormais investis.

\section{Notes}

(1) Enquête sur les Européens, les patrimoines de l'Europe et le patrimoine européen. Ipsos/ministère de la Culture et de la Communication, mars 2007.

(2) Projet de loi sur les musées, La Lettre des Musées de France, n²9, 29 janvier 1993

(3) Grange, S. Le projet de loi Musées de France après le vote des Assemblées : patrimoine ou marché ? Le Journal des Arts, 24 novembre 2001, p. 3.

(4) Audrerie, D. Questions sur le patrimoine. Bordeaux : Confluences, 2003, $119 \mathrm{p}$

(5) Gob, A. Le musée, une institution dépassée ? Paris : Armand Colin, 2010, pp. 49-55.

\section{Bibliographie}

Gob, A. Le musée : une institution dépassée ? Paris : Armand Colin, 2010, $160 \mathrm{p}$.

Greffe, X. et Pflieger, S. La politique culturelle en France. Paris : La Documentation française, 2009, 288 p.

Mairesse, F. Le musée hybride. Paris : La Documentation française, 2010, $208 \mathrm{p}$.

Origet du Cluzeau, C. et Tobelem, J.-M. (dir.) Culture, tourisme et développement : les voies d'un rapprochement. Paris : L'Harmattan, 2009, $271 \mathrm{p}$. 